\title{
Certain Subclass of Analytic Functions Defined by Wanas Operator
}

\author{
Timilehin Gideon Shaba ${ }^{1, *}$, Abbas Kareem Wanas ${ }^{2}$ and \\ Ismaila Omeiza Ibrahim ${ }^{3}$
}

${ }^{1}$ Department of Mathematics, University of Ilorin, P. M. B. 1515, Ilorin, Nigeria e-mail: shabatimilehin@gmail.com

${ }^{2}$ Department of Mathematics, College of Science, University of Al-Qadisiyah, Al Diwaniyah, Al-Qadisiyah, Iraq e-mail: abbas.kareem.w@qu.edu.iq

${ }^{3}$ Department of Mathematical science, University of Maiduguri, Nigeria e-mail: ibrahimismailaomeiza@gmail.com

\begin{abstract}
In present article, we introduce and study a certain family of analytic functions defined by Wanas operator in the open unit disk. We establish some important geometric properties for this family. Further we point out certain special cases for our results.
\end{abstract}

\section{Introduction and Definitions}

Let $\mathfrak{A}$ denote the class of function $\mathfrak{f}(z)$ which are normalized analytic in the open unit disk $\mathfrak{U}=\{z \in \mathcal{C}:|z|<1\}$. Let $\mathfrak{L}(\mathfrak{U})$ be the space of analytic functions in the unit disc $\mathfrak{U}$. Let

$$
\mathfrak{A}_{e}=\left\{\mathfrak{f} \in \mathcal{L}(\mathfrak{U}): \mathfrak{f}(z)=z+\sigma_{e+1} z^{e+1}+\sigma_{e+2} z^{e+2}+\cdots\right\}
$$

with $\mathfrak{A}_{1}=\mathfrak{A}, z \in \mathfrak{U}$ and

$$
\mathcal{L}[\sigma, e]:=\left\{\varphi \in \mathcal{W}(\mathfrak{U}): \varphi(z)=\sigma+\sigma_{e} z^{e}+\sigma_{e+1} z^{e+1}+\cdots\right\}
$$

Received: May 11, 2021; Accepted: June 12, 2021

2010 Mathematics Subject Classification: Primary 30C45; Secondary 30C50.

Keywords and phrases: analytic function, starlike function, convex function, Wanas operator, subordination.

${ }^{*}$ Corresponding author $\quad$ Copyright (C) 2021 Authors 
for $\sigma \in \mathbb{C}$ and $e \in \mathcal{N}$.

We denote by $\mathcal{S}$ subclass of functions which are analytic, univalent in $\mathfrak{U}$ and has the normalization

$$
\mathfrak{f}(z)=z+\sum_{t=2}^{\infty} \sigma_{t+1} z^{t+1}
$$

which implies that

$$
\mathfrak{f}(0)=0, \quad \mathfrak{f}^{\prime}(0)=1 .
$$

A function $\mathfrak{f}(z) \in \mathcal{S}$ is said to be a starlike, convex and turning bounded functions of order $\delta$ which are denoted by $S^{*}(\delta), \mathcal{K}(\delta), \mathcal{R}(\delta) \subset \mathcal{S}$, if the following conditions are satisfied: $\Re\left(\frac{z \mathfrak{f}^{\prime}(z)}{\mathfrak{f}(z)}\right)>\delta, \Re\left(\frac{z f^{\prime \prime}(z)}{\mathfrak{f}^{\prime}(z)}+1\right)>\delta$ and $\Re\left(\mathfrak{f}^{\prime}(z)\right)>\delta$, where $0 \leqq \delta<1$.

Next we recall the definition of subordination. For two functions $h_{1}, h_{2} \in \mathfrak{U}$, we say that $h_{1}$ is subordinated to $h_{2}$ and symbolically written as $h_{1} \prec h_{2}$ if there exists an analytic function $w$ with the property $|w(z)| \leqq|z|$ such that $h_{1}(z)=h_{2}(w(z))$ for $z \in \mathbb{D}$. Further, if $h_{2} \in \mathcal{S}$, then the condition becomes

$$
h_{1} \prec h_{2} \Leftrightarrow h_{1}(0)=h_{2}(0) \text { and } h_{1}(\mathfrak{U}) \subset h_{2}(\mathfrak{U}) .
$$

Wanas [16] in 2019 introduced the following operator, which can also be called (Wanas operator) $\mathfrak{W}_{\beta, n}^{\alpha, \sigma}: \mathfrak{U} \longrightarrow \mathfrak{U}$ defined by

$$
\mathfrak{W}_{\beta, m}^{\mu, \gamma} \mathfrak{f}(z)=z+\sum_{t=2}^{\infty}\left[\amalg_{t}(\gamma, \mu, \beta)\right]^{m} \sigma_{t} z^{t},
$$

where

$$
\amalg_{t}(\gamma, \mu, \beta)=\sum_{a=1}^{\gamma}\left(\begin{array}{l}
\gamma \\
a
\end{array}\right)(-1)^{a+1}\left(\frac{\mu^{a}+t \beta^{a}}{\mu^{a}+\beta^{a}}\right),
$$

$a, m \in \mathcal{N}_{0}, \beta \geqq 0, \mu \in \mathcal{R}$ and $\mu+\beta>0$.

Special cases of this operator can be found in [1, 2, 3, 6, 9, 10, 13, 14, 15]. For more details see [17].

It is readily confirmed from (1.4) that

$$
\begin{aligned}
z\left(\mathfrak{W}_{\beta, m}^{\mu, \gamma} \mathfrak{f}(z)\right)^{\prime}=\left[\sum_{a=1}^{\gamma}\left(\begin{array}{l}
\gamma \\
a
\end{array}\right)(-1)^{a+1}\right. & \left.\left(\left(\frac{\mu}{\beta}\right)^{a}+1\right)\right] \mathfrak{W}_{\beta, m+1}^{\mu, \gamma} \mathfrak{f}(z) \\
& -\left[\sum_{a=1}^{\gamma}\left(\begin{array}{l}
\gamma \\
a
\end{array}\right)(-1)^{a+1}\left(\frac{\mu}{\beta}\right)^{a}\right] \mathfrak{W}_{\beta, m}^{\mu, \gamma} \mathfrak{f}(z) .
\end{aligned}
$$


Lemma 1.1. [8] Let $\phi$ be holomorphic in $\mathfrak{U}$ with $\phi(0)=1$. If

$$
\Re\left(1+\frac{z \phi^{\prime}(z)}{\phi(z)}\right)>\frac{3 \delta-1}{2 \delta},
$$

then $\Re(\phi(z))>\delta$ in $\mathfrak{U}, z \in \mathfrak{U}$ and $\frac{1}{2} \leqq \delta<1$.

\section{Main Result}

Definition 2.1. We say that a function $\mathfrak{f}(z) \in \mathfrak{A}$ is in the class $\mathcal{G}_{\lambda, \delta}^{m}(\gamma, \mu, \beta)$ if

$$
\left|\frac{\mathfrak{W}_{\beta, m+1}^{\mu, \gamma} \mathfrak{f}(z)}{z}\left(\frac{z}{\mathfrak{W}_{\beta, m}^{\mu, \gamma} \mathfrak{f}(z)}\right)^{\lambda}-1\right|<1-\delta \quad(z \in \mathfrak{U}),
$$

where $\beta \geqq 0, \mu \in \mathcal{R}, \mu+\beta>0, \lambda \geq 0, a, m \in \mathfrak{N}_{0}=\{0,1,2,3 \cdots\}$ and $0 \leqq \delta<1$.

Remark 2.2. The family $\mathcal{G}_{\lambda, \delta}^{m}(\gamma, \mu, \beta)$ is a new comprehensive class of holomorphic functions which includes numerous new classes of holomorphic univalent functions as well as some very well-known ones. In place of "equivalence" we are going to take "contained in" as it was discussed in [4], also see [11, 12] for more details. For example,

1. For $m=\mu=0$ and $\gamma=\beta=\lambda=1$, we have the class $\mathcal{G}_{1, \delta}^{0}(1,0,1)$ contained in $S^{*}(\delta)$.

2. For $\mu=0$ and $m=\gamma=\beta=\lambda=1$, we have the class $\mathcal{G}_{1, \delta}^{1}(1,0,1)$ contained in $\mathcal{K}(\delta)$.

3. For $m=\mu=\lambda=0$ and $\gamma=\beta=1$, we have the class $\mathcal{G}_{0, \delta}^{0}(1,0,1)$ contained in $\mathcal{R}(\delta)$.

4. For $\mu=1-\beta$ and $\gamma=1$, we have the class

$$
\mathcal{G}_{\lambda, \delta}^{m}(1,1-\beta, \beta)=\mathcal{G}_{\lambda, \delta}^{m}(\beta)
$$

introduced by Cătaş and Lupas [5]. 
5. For $m=0$, the class

$$
\mathcal{B}(\lambda, \delta)=\left\{\mathfrak{f}(z) \in \mathfrak{A}:\left|\mathfrak{f}^{\prime}(z)\left(\frac{z}{\mathfrak{f}(z)}\right)^{\lambda}-1\right|<1-\delta ; \lambda \geq 0,0 \leqq \delta<1, z \in \mathfrak{U}\right\}
$$

intoduced by Frasin and Jahangiri $[8]$.

6. For $m=0$ and $\lambda=2$, the class

$$
\mathcal{B}(\delta)=\left\{\mathfrak{f}(z) \in \mathfrak{A}:\left|\frac{z^{z} \mathfrak{f}^{\prime}(z)}{\mathfrak{f}^{2}(z)}-1\right|<1-\delta ; 0 \leqq \delta<1, z \in \mathfrak{U}\right\}
$$

intoduced by Frasin and Darus [7].

Theorem 2.3. If for all function $\mathfrak{f}(z) \in \mathfrak{A}, \beta \geqq 0, \mu \in \mathcal{R}, \mu+\beta>0, \lambda \geq 0$, $a, m \in \mathfrak{N}_{0}=\{0,1,2,3 \cdots\}$ and $0 \leqq \delta<1$, we have

$$
\begin{gathered}
\frac{\left[\sum_{a=1}^{\gamma}\left(\begin{array}{l}
\gamma \\
a
\end{array}\right)(-1)^{a+1}\left(\left(\frac{\mu}{\beta}\right)^{a}+1\right)\right] \mathfrak{W}_{\beta, m+2}^{\mu, \gamma} \mathfrak{f}(z)}{\mathfrak{W}_{\beta, m+1}^{\mu, \gamma} \mathfrak{f}(z)} \\
-\frac{\lambda\left[\sum_{a=1}^{\gamma}\left(\begin{array}{l}
\gamma \\
a
\end{array}\right)(-1)^{a+1}\left(\left(\frac{\mu}{\beta}\right)^{a}+1\right)\right] \mathfrak{W}_{\beta, m+1}^{\mu, \gamma} \mathfrak{f}(z)}{\mathfrak{W}_{\beta, m}^{\mu, \gamma} \mathfrak{f}(z)} \\
-(1-\lambda)\left(\sum_{a=1}^{\gamma}\left(\begin{array}{l}
\gamma \\
a
\end{array}\right)(-1)^{a+1}\left(\frac{\mu}{\beta}\right)^{a}+1\right)+1 \prec \varphi z+1, \quad z \in \mathfrak{U},
\end{gathered}
$$

where $\varphi=\frac{-1+3 \delta}{2 \delta}$, then $\mathfrak{f} \in \mathcal{G}_{\lambda, \delta}^{m}(\gamma, \mu, \beta)$.

Proof. Now taking

$$
\phi(z)=\frac{\mathfrak{W}_{\beta, m+1}^{\mu, \gamma} \mathfrak{f}(z)}{z}\left(\frac{z}{\mathfrak{W}_{\beta, m}^{\mu, \gamma} \mathfrak{f}(z)}\right)^{\lambda}
$$

then $\phi(z)$ is holomorphic in $\mathfrak{U}$ with $\phi(0)=1$. With the knowledge of differentiation we have

$$
\log (\phi(z))=\log \left(\mathfrak{W}_{\beta, m+1}^{\mu, \gamma} \mathfrak{f}(z)\right)-\log (z)+\lambda \log (z)-\lambda \log \left(\mathfrak{W}_{\beta, m}^{\mu, \gamma} \mathfrak{f}(z)\right)
$$




$$
\begin{gathered}
\frac{\phi^{\prime}(z)}{\phi(z)}=\frac{\left(\mathfrak{W}_{\beta, m+1}^{\mu, \gamma} \mathfrak{f}(z)\right)^{\prime}}{\mathfrak{W}_{\beta, m+1}^{\mu, \gamma} \mathfrak{f}(z)}-\frac{\phi\left(\mathfrak{W}_{\beta, m}^{\mu, \gamma} \mathfrak{f}(z)\right)^{\prime}}{\mathfrak{W}_{\beta, m}^{\mu, \gamma} \mathfrak{f}(z)}-\left(\frac{1-\phi}{z}\right) \\
\frac{\phi^{\prime}(z)}{\phi(z)}=\frac{\left[\sum_{a=1}^{\gamma}\left(\begin{array}{l}
\gamma \\
a
\end{array}\right)(-1)^{a+1}\left(\left(\frac{\mu}{\beta}\right)^{a}+1\right)\right] \mathfrak{W}_{\beta, m+2}^{\mu, \gamma} \mathfrak{f}(z)}{\mathfrak{W}_{\beta, m+1}^{\mu, \gamma} \mathfrak{f}(z)} \\
-\frac{\sum_{a=1}^{\gamma}\left(\begin{array}{l}
\gamma \\
a
\end{array}\right)(-1)^{a+1}\left(\frac{\mu}{\beta}\right)^{a}}{z}+\frac{\lambda \sum_{a=1}^{\gamma}\left(\begin{array}{c}
\gamma \\
a
\end{array}\right)(-1)^{a+1}\left(\frac{\mu}{\beta}\right)^{a}}{z} \\
-\frac{\lambda\left[\sum_{a=1}^{\gamma}\left(\begin{array}{l}
\gamma \\
a
\end{array}\right)(-1)^{a+1}\left(\left(\frac{\mu}{\beta}\right)^{a}+1\right)\right] \mathfrak{W}_{\beta, m+1}^{\mu, \gamma} \mathfrak{f}(z)}{z \mathfrak{W}_{\beta, m}^{\mu, \gamma} \mathfrak{f}(z)}-\left(\frac{1-\phi}{z}\right)
\end{gathered}
$$

multiplying throughout by $z$, yields

$$
\begin{aligned}
& \frac{z \phi^{\prime}(z)}{\phi(z)}=\frac{\left[\sum_{a=1}^{\gamma}\left(\begin{array}{l}
\gamma \\
a
\end{array}\right)(-1)^{a+1}\left(\left(\frac{\mu}{\beta}\right)^{a}+1\right)\right] \mathfrak{W}_{\beta, m+2}^{\mu, \gamma} \mathfrak{f}(z)}{\mathfrak{W}_{\beta, m+1}^{\mu, \gamma} \mathfrak{f}(z)}-\frac{\lambda\left[\sum_{a=1}^{\gamma}\left(\begin{array}{l}
\gamma \\
a
\end{array}\right)(-1)^{a+1}\left(\left(\frac{\mu}{\beta}\right)^{a}+1\right)\right] \mathfrak{W}_{\beta, m+1}^{\mu, \gamma} \mathfrak{f}(z)}{\mathfrak{W}_{\beta, m}^{\mu, \gamma} \mathfrak{f}(z)} \\
&-(1-\lambda)\left(\sum_{a=1}^{\gamma}\left(\begin{array}{l}
\gamma \\
a
\end{array}\right)(-1)^{a+1}\left(\frac{\mu}{\beta}\right)^{a}+1\right) .
\end{aligned}
$$

Applying (2.1), we have

$$
\Re\left(1+\frac{z \phi^{\prime}(z)}{\phi(z)}\right)>\frac{-1+3 \delta}{2 \delta} .
$$

Thus, by the application of Lemma 1.1, we have

$$
\Re\left(\frac{\mathfrak{W}_{\beta, m+1}^{\mu, \gamma} \mathfrak{f}(z)}{z}\left(\frac{z}{\mathfrak{W}_{\beta, m}^{\mu, \gamma} \mathfrak{f}(z)}\right)^{\lambda}\right)>\delta .
$$

Hence, $\mathfrak{f} \in \mathcal{G}_{\lambda, \delta}^{m}(\gamma, \mu, \beta)$, by the reason of Definition 2.1 . 
Varying the parameters of the above theorem gives the following corollaries.

Corollary 2.4. Suppose $\mathfrak{f}(z) \in \mathfrak{A}$ and

$$
\Re\left(\frac{z \mathfrak{f}^{\prime \prime}(z)}{\mathfrak{f}^{\prime}(z)}+1\right)>\frac{1}{2}, \quad z \in \mathfrak{U},
$$

then

$$
\Re\left(\mathfrak{f}^{\prime}(z)\right)>\frac{1}{2}, \quad z \in \mathfrak{U} .
$$

We can as well say that, if the function $\mathfrak{f}(z)$ is convex of order $1 / 2$, then $\mathfrak{f}(z) \in$ $\mathcal{G}_{0, \delta}^{0}(1,0,1)$ contained in $\mathcal{R}(1 / 2)$.

Corollary 2.5. Suppose $\mathfrak{f}(z) \in \mathfrak{A}$ and

$$
\Re\left(-\frac{z \mathfrak{f}^{\prime \prime}(z)}{\mathfrak{f}^{\prime}(z)}+\frac{z\left(z \mathfrak{f}^{\prime \prime \prime}(z)+\mathfrak{f}^{\prime \prime}(z)\right)}{z \mathfrak{f}^{\prime \prime}(z)+\mathfrak{f}^{\prime}(z)}\right)>-\frac{1}{2} \quad z \in \mathfrak{U},
$$

then $\mathfrak{f}(z) \in \mathcal{G}_{1,1 / 2}^{1}(1,0,1)$, therefore

$$
\Re\left(\frac{z \mathfrak{f}^{\prime \prime}(z)}{\mathfrak{f}^{\prime}(z)}+1\right)>\frac{1}{2}, \quad z \in \mathfrak{U} .
$$

Which implies that $\mathfrak{f}(z)$ is convex of order $1 / 2$.

Corollary 2.6. Suppose $\mathfrak{f}(z) \in \mathfrak{A}$ and

$$
\Re\left(-\frac{z \mathfrak{f}^{\prime}(z)}{\mathfrak{f}^{\prime}(z)}+\frac{\mathfrak{f}(z)+3 z \mathfrak{f}^{\prime}(z)+z^{2} \mathfrak{f}^{\prime \prime}(z)}{z \mathfrak{f}^{\prime}(z)+\mathfrak{f}^{\prime}(z)}\right)>\frac{1}{2}, \quad z \in \mathfrak{U},
$$

then

$$
\Re\left(\frac{z \mathfrak{f}^{\prime}(z)}{\mathfrak{f}(z)}\right)>0, \quad z \in \mathfrak{U} .
$$

Which implies that $\mathfrak{f}(z)$ is a starlike function.

Corollary 2.7. [5] Suppose $\mathfrak{f}(z) \in \mathfrak{A}$ and

$$
\Re\left(\frac{5 z \mathfrak{f}^{\prime}(z)-\mathfrak{f}(z)+z^{2} \mathfrak{f}^{\prime \prime}(z)}{z \mathfrak{f}(z)+\mathfrak{f}(z)}\right)>-\frac{1}{2}, \quad z \in \mathfrak{U},
$$

then

$$
\Re\left(-2+\frac{\mathfrak{f}(z)}{z}+\mathfrak{f}^{\prime}(z)\right)>2, \quad z \in \mathfrak{U} .
$$




\section{References}

[1] J. W. Alexander, Functions which map the interior of the unit circle upon simple region, Ann. of Math. (2) 17 (1915), 12-22. https://doi.org/10.2307/2007212

[2] F. M. Al-Oboudi, On univalent functions defined by a generalized Sălăgean operator, Int. J. Math. Math. Sci. 27 (2004), 1429-1436.

https://doi.org/10.1155/S0161171204108090

[3] S. D. Bernardi, Convex and starlike univalent functions, Trans. Amer. Math. Soc. 135 (1969), 429-446. https://doi.org/10.1090/S0002-9947-1969-0232920-2

[4] K. O. Babalola, Combinations of geometric expressions implying schlichtness, An. Univ. Oradea Fasc. Mat. 21 (2014), 91-94.

[5] A. Cătaş and A. A. Lupas, On a subclass of analytic functions defined by a generalized Sălăgean operator, ROMAI J. 4(2) (2008), 57-60.

[6] N. E. Cho and H. M. Srivastava, Argument estimates of certain analytic functions defined by a class of multiplier transformations, Math. Comput. Modelling 37 (2003), 39-49. https://doi.org/10.1016/S0895-7177(03)80004-3

[7] B. A. Frasin and M. Darus, On certain analytic univalent functions, Int. J. Math. Math. Sci. 25 (2001), 305-310. https://doi.org/10.1155/S0161171201004781

[8] B. A. Frasin and J.M. Jahangiri, A new and comprehensive class of analytic functions, An. Univ. Oradea Fasc. Mat. 15 (2008), 59-62.

[9] I. B. Jung, Y. C. Kim and H. M. Srivastava, The Hardy space of analytic functions associated with certain one-parameter families of integral operators, J. Math. Anal. Appl. 176 (1993), 138-147. https://doi.org/10.1006/jmaa.1993.1204

[10] G. Şt. Sălăgean, Subclasses of univalent functions, Lecture Notes in Math., Springer Verlag, Berlin 1013 (1983), 362-372. https://doi.org/10.1007/BFb0066543

[11] T. G. Shaba, A. A. Ibrahim, L. O. Ahmed, On a comprehensive class of $p$-valent functions defined by generalized Al-Oboudi differential operator, Advances in Mathematics: Scientific Journal 9(12) (2020), 10633-10638.

https://doi.org/10.37418/amsj.9.12.48 
[12] T. G. Shaba, On certain analytic functions defined by Frasin differential operator, Int. J. Open Problems Complex Analysis 12(3) (2020), 13-18.

[13] H. M. Srivastava and A. A. Attiya, An integral operator associated with the Hurwitz-Lerch zeta function and differential subordination, Integral Transforms Spec. Funct. 18 (2007), 207-216. https://doi.org/10.1080/10652460701208577

[14] S. R. Swamy, Inclusion properties of certain subclasses of analytic functions, Int. Math. Forum 7 (2012), 1751-1760.

[15] B. A. Uralegaddi and C. Somanatha, Certain classes of univalent functions, in: Current Topics in Analytic Function Theory, Edited by H. M. Srivastava and S. Own, 371-374, World Scientific, Singapore, 1992. https://doi.org/10.1142/9789814355896_0032

[16] A. K. Wanas, New differential operator for holomorphic functions, Earthline J. Math. Sci. 2 (2019), 527-537.https://doi.org/10.34198/ejms.2219.527537

[17] A. K. Wanas and G. Murugusundaramoorthy, Differential sandwich results for Wanas operator of analytic functions, Math. Morav. 24 (2020), 17-28. https://doi.org/10.5937/MatMor2001017K

This is an open access article distributed under the terms of the Creative Commons Attribution License (http://creativecommons.org/licenses/by/4.0/), which permits unrestricted, use, distribution and reproduction in any medium, or format for any purpose, even commercially provided the work is properly cited. 